\title{
Habilidades emprendedoras: importancia, evolución y sub temas emergentes. Una revisión de literatura
}

\section{Entrepreneurial skills: importance, evolution and emerging sub-themes. A literature review}

\section{Resumen}

Artículo de revisión.

Fecha de recepción: 10/07/2020

Fecha de devolución: 20/09/2020

Fecha de aceptación: 10/10/2020

Fecha de publicación: 17/10/2020

\section{Carolina Ocampo Sanabria}

Universidad Católica Luis Amigó

Manizales, Risaralda (Colombia)

carolina.bricam@gmail.com

\section{Yurani López Espitia}

Universidad Católica Luis Amigó

Manizales, Risaralda (Colombia)

yurani.lopezes@amigo.edu.co

Para citar este artículo:

Ocampo, C. \& López, Y. (2021). Habilidades emprendedoras: importancia, evolución y sub temas emergentes. Una revisión de literatura. Económicas CUC, 42(1), 189-207. DOI: https:// doi.org/10.17981/econcuc.42.1.2021.Org.4
El emprendimiento ha sido en los últimos años un pilar para la economía mundial y en este entendido el presente artículo plantea una base conceptual que aborda la temática referente a las habilidades y al emprendimiento, dentro de este estudio se analiza principalmente el concepto del emprendimiento y se identifican algunas habilidades que son necesarias para los emprendedores que pretenden alcanzar objetivos que se fijan desde una etapa temprana. El enfoque de esta investigación es realizar una revisión de literatura sobre la importancia que tiene el fenómeno del emprendimiento, el cual es el propósito central del artículo; de igual manera enfatizar acerca de las habilidades, ya que se convierten en un pilar pertinente dentro de este contexto, porque son una base que permiten abordar posibles problemas permanentes y de magnitudes diferentes que surgen mediante procesos de revoluciones. Para cumplir con esta estructura, se hizo una consulta en Web of Science y posteriormente se hizo una selección de los documentos más importantes a través de la plataforma Tree of Science y, al final, se efectúa un análisis de perspectivas o subtemas que especifican las habilidades emprendedoras. Dentro de los hallazgos se encuentra que el concepto de emprendedores aclara que las habilidades sociales de los empresarios son competencias específicas que ayudan a la interacción y generación de contactos personales de manera socioproductiva con otros individuos. Se concluye que el emprendimiento o espíritu empresarial son un método de acción humana.

Palabras clave: Habilidades emprendedoras; emprendimiento; revisión documental; investigación; estado del arte; habilidades sociales; habilidades emprendedoras en la economía; habilidades equilibradas

\begin{abstract}
Entrepreneurship has been in recent years a pillar for the world economy and in this understanding the present this article provides a conceptual basis that addresses the topic of skills and entrepreneurship. This study mainly analyses the concept of entrepreneurship and identifies some skills that are necessary for entrepreneurs who aim to achieve goals that are set from an early stage. The focus of this research in to conduct a literature review on the importance of the phenomenon of entrepreneurship, which is the central purpose of the article; in the same way emphasize about skills, since they become a relevant pillar within this context because they are a basis for addressing potential permanent problems and different magnitudes that arise through revolution processes. To comply with this structure, a consultation was made in Web of Science and subsequently a selection of the most important documents was made through the Tree of Science platform and, in the end, an analysis of perspectives or subtopics was carried out specifying entrepreneurial skills. Among the findings, it is found that the concept of entrepreneurs clarifies that the social skills of entrepreneurs are specific competencies that help the interaction and generation of personal contacts in a socio-productive way with other individuals. It is concluded that entrepreneurship or entrepreneurship are a method of human action. Keywords: Entrepreneurial skills; entrepreneurship; documentary review; research; state of the art; social skills; entrepreneurial skills in economics; balanced skills
\end{abstract}




\section{INTRODUCCIÓN}

El emprendimiento generalmente surge por una necesidad o una oportunidad, así lo indican los recientes desarrollos dedicados al plan de estudio del emprendimiento han sido notables, porque este campo ha surgido en las últimas dos décadas como posiblemente la fuerza económica más potente que el mundo haya experimentado, (Kuratko, 2005). Por consiguiente, algunas características que tiene el emprendimiento son: primero, es un término que se refiere a alguien que crea y luego organiza y opera una nueva empresa comercial; y segundo, alguien innovador como el que transforma invenciones e ideas en entidades económicamente viable (Baumol, 1993; Henríquez, Rada \& Torrenegra, 2016). El enfoque del emprendimiento conserva una importancia ya que tiene que ver con el espíritu empresarial que se enfoca con la generación de una organización u empresa lucrativa (Sabar \& Pagis, 2015).

Krieger, Block y Stuetzer (2018) estudiaron la teoría de Lazear acerca del equilibrio en las habilidades o más conocida como "jack-of-all-trades", el cual significa que los emprendedores son generalistas y empleados especialistas; este estudio mostró que el equilibrio en habilidades son un factor importante para que una persona decida volverse emprendedora. Claramente las habilidades son importantes en la creación y gestión de una empresa, sin embargo, las destrezas para emprender se manifiestan de manera diferente en las distintas fases por las que se debe pasar, es decir, etapas que muestran la importancia, complejidad y cambio de las habilidades requeridas en cada aspecto; en otras palabras, se propone que la aplicación de diferentes habilidades es necesaria dependiendo de la etapa y el contexto del emprendimiento (Henríquez et al., 2016; Mamabolo \& Myres, 2020).

De acuerdo con el propósito del artículo, se ha enfatizado en distintos estudios que el emprendimiento se ha vuelto cada vez más importante en todo el mundo porque es una conducta que genera un impacto positivo en el empleo, la productividad, la innovación y el crecimiento económico. En consecuencia, las habilidades son un complemento importante y se deben tener en cuenta, ya que influyen en el logro del emprendimiento, básicamente un conjunto de habilidades técnicas contribuye a convertir un individuo en un emprendedor exitoso (Sousa, 2018).

Para cumplir con el propósito establecido previamente, se hizo uso de una herramienta web que simplificó sumamente la investigación del tema correspondiente. Las consultas se hicieron mediante la herramienta de Tree of Science (ToS) que clasifica los artículos pertinentes y necesarios para realizar la estructuración de este artículo. De esta manera la producción científica puede visualizarse en forma de árbol lo que permite entender mejor la influencia actual del tema, con una raíz donde se encuentran los clásicos, en el tronco están los estructurales y en las hojas son los recientes, y de esta manera se determinan subcategorías mediante un análisis de co-citaciones. Esta herramienta de ToS fue esencial para la ampliación de información que se adecua dentro de este contexto. 


\section{MÉTODOLOGÍA}

La metodología se estructura en tres partes. El primer paso muestra la importancia del tema de investigación a partir de análisis cienciométricos como la producción científica anual. El segundo paso muestra la forma como se determinó la evolución del tema a partir del algoritmo de Tree of Science. Finalmente, en el paso tres se explica la forma como se construyó la red de citaciones para generar las diferentes subáreas del tema. En la Figura 1 se muestra gráficamente las herramientas que permitieron organizar la temática de este estudio.

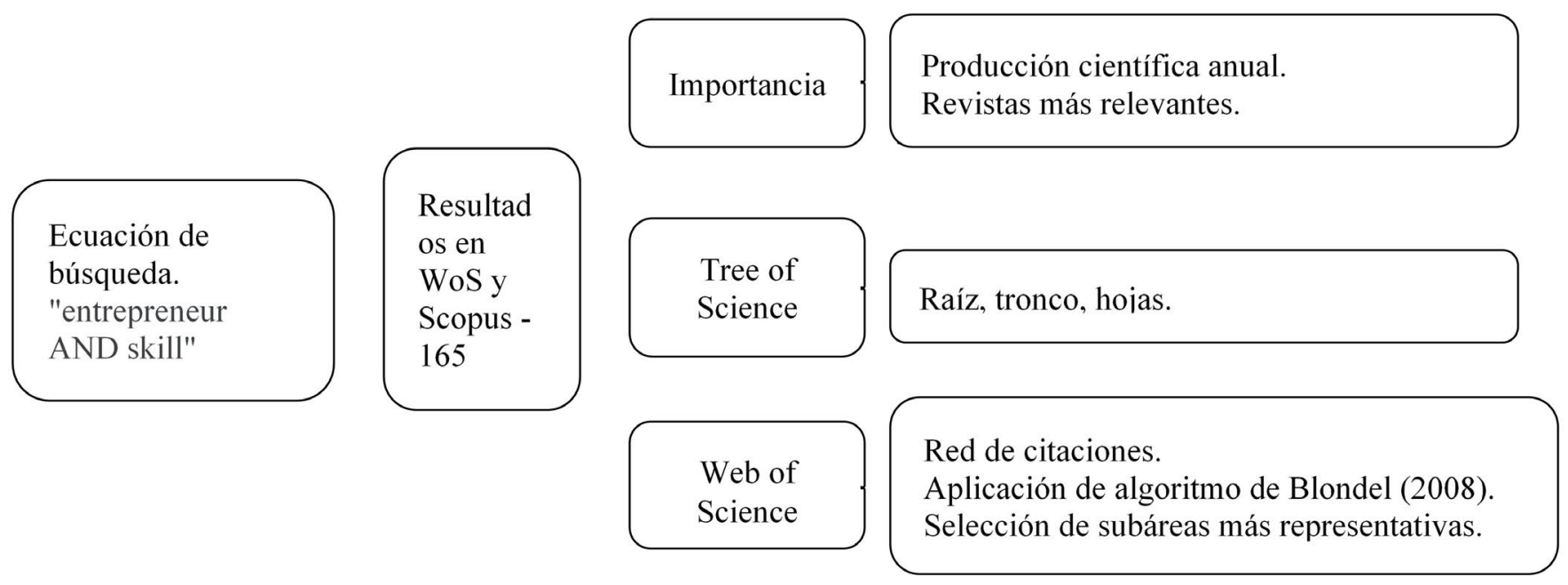

Figura 1. Proceso general para identificar la importancia, la evolución y las subáreas de las habilidades emprendedoras. Fuente: Elaboración propia.

\section{Paso 1: Importancia}

Se realizó una consulta en Web of Science (WoS) con el criterio de búsqueda en título "entrepreneur" y "skill" para determinar los artículos que trataran sobre habilidades en el emprendimiento; los resultados fueron 165 registros. Esta misma consulta se realizó en Scopus para comparar la producción científica sobre el tema en las dos bases de datos. Con estos resultados, se identificó la evolución y el avance del estudio de este tema a partir de la producción científica anual y la calidad de las revistas en las que se publica en esta área.

\section{Paso 2: Tree of science}

La información obtenida anteriormente fue resultado de ingresar a la herramienta web de Tree of Science (ToS) (Robledo, Duque-Méndez \& Zuluaga-Giraldo, 2014), la cual sirvió para facilitar la búsqueda de documentos que fueron base para desarrollar la temática correspondiente a las habilidades emprendedoras. ToS ha sido utilizado en diferentes áreas como la psicología (Landínez, Robledo \& Montoya, 2019; Landínez \& Montoya, 2019), responsabilidad social universitaria (Duque \& Cervantes-Cer- 
vantes, 2019), branding corporativo (Buitrago, Duque \& Robledo, 2019) y decisiones éticas en las organizaciones (Díez-Gómez, Guillén y Rodríguez, 2019). Por lo tanto, es una metodología aceptada en el ámbito científico. ToS utiliza la teoría de grafos para identificar los artículos más importantes de acuerdo a su posición dentro de la red para finalmente ubicarlos en raíz, tronco y hojas (Robledo-Giraldo et al., 2013)

\section{Paso 3: Análisis de Citaciones}

Para identificar las subáreas, se creó una red de citaciones utilizando la metodología propuesta por Zuluaga et al. (2016). Después se aplicó un algoritmo de clusterización para identificar las tres áreas más grandes de las habilidades emprendedoras (Blondel, Guillaume, Lambiotte \& Lefebvre, 2008). Una vez identificadas, se realizó un web scraping con los DOIs de los artículos para extraer los títulos y realizar una minería de texto para identificar los temas de cada subárea. La red final y la nube de palabras se muestran en la Figura 3.

\section{RESULTADOS}

\section{Importancia}

El propósito central del artículo se enfoca en mostrar la importancia que se le atribuye al emprendimiento y adicionalmente explorar las habilidades que permiten impulsar la actividad emprendedora en cualquier contexto. El emprendimiento es un campo que ha tenido diversos desarrollos y una sustancial trascendencia mediante estudios e investigaciones detalladas que aportan conocimientos diferentes.

\section{Producción científica anual en WoS y Scopus}

- WoS - Scopus

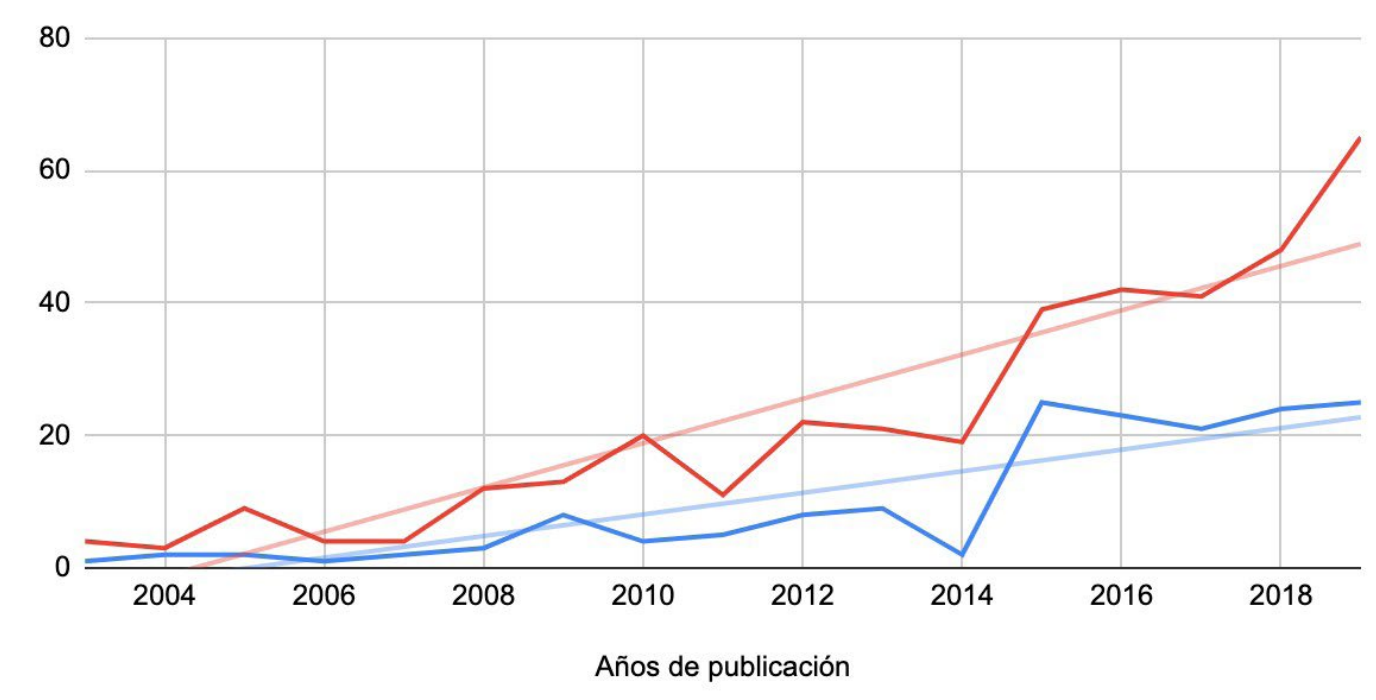

Figura 2. Producción científica anual acerca de habilidades emprendedoras. Fuente: Elaboración propia. 
La Figura 2 muestra la evolución de estudios y la producción científica que se han hecho respecto al tema. De igual manera el gráfico presenta la evidencia de que existen revisiones que se han realizado de forma continua dado a su curva de ascenso y que implican ser valiosos, dado a la alta importancia que se ha atribuido a las habilidades emprendedoras.

El área del emprendimiento y las habilidades ha alcanzado un nivel de importancia amplio en diferentes campos, que es necesario hacer una revisión detallada, para ello se tendrán en cuenta las perspectivas de este campo de estudio en la actualidad y, de igual manera, se incluirá un análisis de red de apoyo con otros artículos relacionados con el tema, que sirvieron de fuente para la estructuración de esta revisión de emprendimiento y habilidades para cualquier ámbito.

En la Tabla 1 se relacionan las 5 revistas con mayor número de publicaciones en el tema específico del emprendimiento y habilidades. Cuatro de ellas están en el cuartil 1 (Q1), siendo la más importante "Technovation", que cuenta con el más alto factor de impacto.

TABla 1.

Revistas más relevantes en habilidades de emprendimiento

\begin{tabular}{|c|c|c|c|}
\hline Revistas & Artículos & Cuartil & Factor de Impacto* \\
\hline Small Business Economics & 9 & Q1 & 1.91 \\
\hline $\begin{array}{l}\text { International Entrepreneurship } \\
\text { and Management Journal }\end{array}$ & 4 & Q1 & 1.05 \\
\hline $\begin{array}{l}\text { International Journal of } \\
\text { Entrepreneurial Behaviour and } \\
\text { Research }\end{array}$ & 3 & Q1 & 0.79 \\
\hline $\begin{array}{l}\text { Journal of Home Economics } \\
\text { Research }\end{array}$ & 3 & $\mathrm{Q} 4^{* *}$ & $0.051^{* *}$ \\
\hline Technovation & 3 & $\mathrm{Q} 1$ & 2.3 \\
\hline
\end{tabular}

Fuente: ${ }^{*}$ scimagojr.com ${ }^{* *}$ Web of Science.

\section{Tree of Science}

Al ser identificados los artículos más importantes y que aportaron mayormente al orden de esta investigación, se permitió dar una visión general del tema propuesto. De igual manera son documentos que dan un soporte a la teoría del emprendimiento y las habilidades como fuente para crear un espíritu emprendedor, y de esa manera dar como resultado un ambiente de éxito y progreso. Dentro de los resultados se confirmó que las habilidades necesarias en las personas deben ser compatibles con su capacidad de desarrollarlas, para tener un mejor desempeño y desenvolvimiento en un entorno social o empresarial y para permitir de manera fácil la solución de problemas, la comunicación, el trabajo en equipo entre otras destrezas (Sahlberg, 2006). 


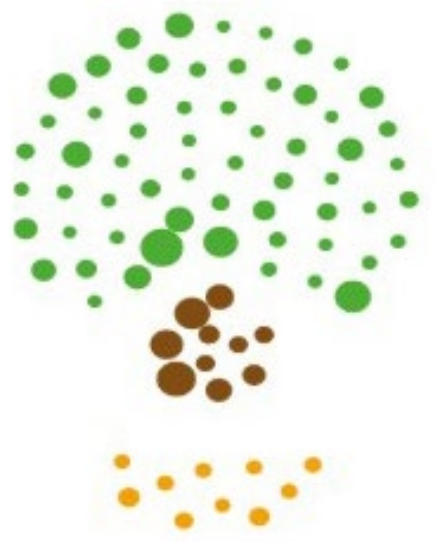

Figura 3. Árbol de la ciencia de habilidades emprendedoras.

Fuente: Elaboración propia.

La anterior visualización (Figura 3) permite tener una visión de la estructura de algunos de los artículos seleccionados más importantes en cada categoría según el algoritmo empleado por la plataforma ToS.

TABLA 2.

Documentos principales

\begin{tabular}{|c|c|c|}
\hline Raíz & Tronco & Hojas \\
\hline $\begin{array}{l}\text { Entrepreneurs' Social Skills } \\
\text { and New Venture Performance: } \\
\text { Mediating Mechanisms and } \\
\text { Cultural Generality (Baron \& } \\
\text { Tang, 2009). }\end{array}$ & $\begin{array}{l}\text { Balanced skills among } \\
\text { nascent entrepreneurs } \\
\text { (Stuetzer, Obschonka \& Schmitt- } \\
\text { Rodermund, 2013b). }\end{array}$ & $\begin{array}{l}\text { Low Returns in R\&D } \\
\text { due to the Lack of } \\
\text { Entrepreneurial Skills } \\
\text { (Michelacci, 2003). }\end{array}$ \\
\hline Entrepreneurship (Lazear, 2005). & $\begin{array}{l}\text { Balanced Skills and } \\
\text { Entrepreneurship (Lazear, 2004). }\end{array}$ & $\begin{array}{l}\text { Entrepreneurship: skills } \\
\text { and financing (Balmaceda, } \\
\text { 2018). }\end{array}$ \\
\hline $\begin{array}{l}\text { Are nascent entrepreneurs 'Jacks- } \\
\text { of-all-trades'? A test of Lazear's } \\
\text { theory of entrepreneurship with } \\
\text { German data (Wagner, 2006). }\end{array}$ & $\begin{array}{l}\text { The impact of skills, working } \\
\text { time allocation and peer effects } \\
\text { on the entrepreneurial intentions } \\
\text { of scientists (Moog, Werner, } \\
\text { Houweling \& Backes-Gellner, } \\
\text { 2015). }\end{array}$ & $\begin{array}{l}\text { Risk, balanced skills and } \\
\text { entrepreneur (Hsieh, } \\
\text { Parker \& Van Praagship, } \\
\text { 2017). }\end{array}$ \\
\hline $\begin{array}{l}\text { Beyond social capital: How social } \\
\text { skills can enhance entrepreneurs' } \\
\text { success (Baron \& Markman, } \\
\text { 2000). }\end{array}$ & $\begin{array}{l}\text { Entrepreneur political skill } \\
\text { and new venture performance: } \\
\text { Extending the social competence } \\
\text { perspective (Tocher, Oswald, } \\
\text { Shook \& Adams, 2012). }\end{array}$ & $\begin{array}{l}\text { A study on the political skill } \\
\text { of Korean entrepreneurs } \\
\text { contributing to social } \\
\text { network guarantee (Zhan \& } \\
\text { Kim,2015). }\end{array}$ \\
\hline
\end{tabular}

Fuente: Elaboración propia.

Para sustentar con mayor integralidad los resultados de este artículo, se tuvieron en cuenta 59 casos de estudio enfocados en fundamentar el tema de emprendimiento y habilidades. Estos fueron tomados de la estructura del árbol generado con la herramienta de Tree of Science. Puntualmente se relacionan algunos artículos de cada parte del árbol: la raíz, el tronco y las hojas, con el objetivo de ilustrar el tema a partir de investigaciones que soporten los conceptos de las habilidades para emprender. 
Los artículos que se tuvieron en cuenta, ubicados en la raíz del árbol (Tabla 2), principalmente se enfocan en que el término de emprendedor en relación con el término de empresario, destacan las similitudes que tienen ambas palabras, generalmente, estos artículos hacen mayor énfasis en la palabra 'empresario'. Por ejemplo, Chen, Greene y Crick (1998) proponen un constructo de autoeficacia empresarial, que consiste en una fuerza de la creencia que tiene una persona para sentirse capaz de realizar con éxito diversos roles y tareas que involucren e influyan una iniciativa empresarial.

Por otro lado, Hartog, Van Praag y Van Der Sluis (2010) generan un punto de vista que se enfoca en aportar tipos de habilidades que particularmente se conocen como habilidades cognitivas, que son una fuente para crecer e impulsar el espíritu empresarial. Por lo tanto, tales habilidades se identifican como capacidades verbales, capacidades sociales y matemáticas que se practican esencialmente para la generación de ingresos o salarios, incluso el equilibrio en el desarrollo de estas habilidades mencionadas influye en las ganancias de los empresarios.

Finalmente, Davidsson y Honig (2003) hicieron un estudio en donde examinan la iniciativa de emprendimiento incipiente, es decir, las primeras etapas tempranas de la actividad emprendedora; y analizan que la fase preliminar de un proceso emprendedor comprende diversas situaciones, entre ellas están la sugerencia de conservar, buscar y desarrollar activamente las relaciones sociales, de igual manera adaptan esta etapa preliminar como la forma en que se detectan las oportunidades y se actúa sobre ellas. Dentro de este estudio se relaciona el capital humano como un modo de aumentar habilidades cognitivas, lo que lleva a una actividad potencial más productiva y eficiente, y el capital social como la capacidad de los actores para extraer beneficios de sus estructuras sociales.

De este grupo de trabajos se puede concluir que el empresario se caracteriza por atravesar una etapa preliminar en donde lleva a cabo un emprendimiento mediante el descubrimiento de oportunidades, las cuales son claves para potenciar el éxito con la ayuda de diversas habilidades como por ejemplo verbales, matemáticas y sociales. Y que durante el proceso naciente se involucran una serie de aspectos relacionados al capital social y humano, lo que significa que son influencias teóricas que aportan resultados al proceso.

En cuanto a los artículos que se encuentran en el tronco, estos sirven para darle forma a la teoría. Son artículos con un sentido más claro y más dirigidos hablar acerca de las habilidades que son necesarias para operar en una actividad específica, ya sea empresarial o emprendedora. Uno de los primeros artículos es el de Oosterbeek, Van Praag y Ijsselstein (2010), el cual describe el impacto de la educación empresarial en las habilidades y la motivación empresarial de estudiantes universitarios. El investigador aplicó un instrumento entre estudiantes para medir las habilidades empresariales autoevaluadas entre ellos, y el nivel de iniciativa para convertirse en empresarios; sin embargo, los resultados fueron insignificantes e incluso negativos.

De igual manera, Stuetzer, Obschonka, Davidsson y Schmitt-Rodermund (2013a) comparten información sobre las fuentes de dónde vienen las habilidades para 
implementarlas en un entorno empresarial y principalmente encontraron que la mayoría de habilidades que adquiere una persona, son por un conjunto variado de experiencias laborales. Finalmente, Tocher et al. (2012) realizaron una investigación que evalúa específicamente la capacidad de un individuo para influir en las acciones de otras personas dentro de un entorno empresarial, y que por lo tanto se asocia positivamente con el desempeño de una nueva empresa.

Se concluye entonces, que las habilidades y la motivación empresarial no son comunes en la población en general, que muchas habilidades de emprendimiento provienen más de la experiencia laboral que del capital humano en su visión tradicional y que las habilidades representan la capacidad de influir en otras personas que propician el éxito en el campo del emprendimiento.

Respecto a los artículos obtenidos de la parte de las hojas, estos textos contienen información desde una perspectiva más global, es decir, abarcan estudios centrados en escenarios internacionales y al mismo tiempo se concentran en evaluar las prácticas de las habilidades dentro de una comunidad con condiciones sociales de bajos ingresos. Referente a esto, Brixiova y Égert (2017) proponen que una economía para implementar un equilibrio productivo, principalmente se deben de abordar las faltas de habilidades y a partir de ahí, crear una mejor educación para compensar los desajustes en las habilidades para encauzar un mejor entorno empresarial y por ende económico.

Otro de los hallazgos que hace referencia a la escasez de habilidades lo proporciona Brixiová, Ncube y Bicaba (2015), quienes investigaron sobre una notoria ausencia de habilidades empresariales que conllevan a determinar que hay una reducida población de jóvenes con iniciativas emprendedoras, y que por lo tanto los jóvenes deben de enfrentarse a altos costos de búsqueda de oportunidades, lo que impide la creación de empresas productivas. Por último, Hodges et al. (2015) reportan las necesidades que surgen en los profesionales por obtener conocimientos empresariales y habilidades necesarias adaptadas a la creación de organizaciones de intenciones lucrativas.

Aquí, podemos concluir que un entorno con pocas oportunidades y baja educación en habilidades emprendedoras hace más difícil el surgimiento de emprendedores exitosos; también la búsqueda de opciones y el desarrollo de un nuevo emprendimiento están casi fuera de alcance. La iniciativa de formar un futuro emprendedor con las habilidades deseables y necesarias puede ser positiva para la creación de empresas y por ende el crecimiento económico.

\section{Sub temas de las habilidades emprendedoras}

Los resultados del análisis de citaciones arrojaron 12 subáreas; sin embargo, se escogieron las tres más grandes, es decir, que tuvieran la mayor cantidad de artículos. En total, los tres subtemas representan el 35,74\% del total de artículos de la red de citaciones, solo el 25\% de los grupos escogidos equivalen a casi el $40 \%$ del total de artículos. En la Figura 4 se muestra la red de citaciones de los tres subtemas con la nube de palabras de cada uno. 


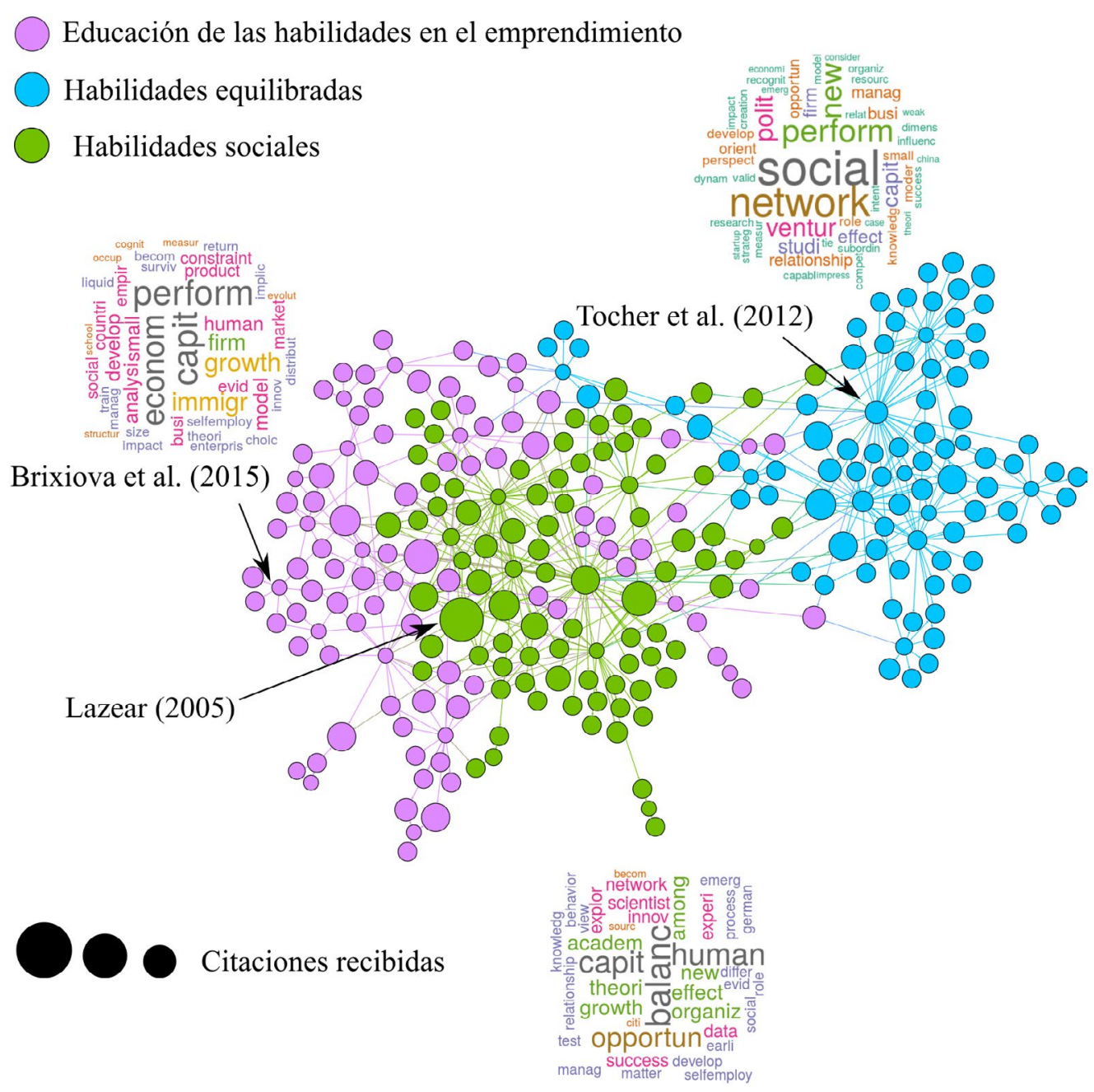

Figura 4. Red de co-citaciones del Emprendimiento y Habilidades. Fuente: Elaboración propia.

\section{- Sub tema 1: Impacto de las habilidades emprendedoras en la economía}

El primer enfoque muestra una red que tiene 98 nodos (artículos). Dentro de este subtema los artículos que fueron objeto de análisis son 10 nodos, los cuales se seleccionaron por el motivo de que son documentos que aportan estudios referentes al ámbito económico, relacionando la actividad emprendedora con el alcance de habilidades que conducen a un desarrollo económico o financiero en un país o una corporación.

Respecto a este subtema se consideran las investigaciones que soportan la teoría del impacto de las habilidades del emprendimiento en el área económica. En términos generales los artículos tenidos en cuenta plantean la importancia del tema como lo hace Bernat et al. (2009), con un aporte muy amplio sobre una investigación de habilidades de emprendimiento en estudiantes de diferentes países particularmente de Europa. Puntualmente en una encuesta realizada en varios países europeos para determinar cómo el desarrollo dinámico del emprendimiento ha logrado transformar sistemáticamente a dichas naciones. En la investigación se desarrolla un concepto de espíritu empresarial que la define como la realización de una actividad económica, también es entendida como una conducción efectiva en condiciones de riesgo y competencia y lo más importante es una cualidad inherente de las sociedades. 
Es importante detallar los diversos panoramas que surgen entorno al campo del emprendimiento. Reynolds et al. (2005) y Sarasvathy y Venkataraman (2011) proponen un argumento referente a que existe un "método emprendedor" como un ámbito pertinente a los asuntos humanos, porque básicamente el fundamento de este método consiste en enseñar emprendimiento no solo para emprendedores sino para todos, como una habilidad necesaria y útil.

Reynolds et al. (2005) da sentido a este argumento planteando un programa de investigación que se centra en un importante impulsor de crecimiento económico involucrando el espíritu empresarial y reconoce que el emprendimiento es una de las fuerzas más importantes porque dan forma a los cambios en el panorama económico. Finalmente, Audretsch, Bönte y Keilbach (2008) resaltan la importancia de la innovación como habilidad para generar impacto positivo en la actividad empresarial y también plantean que el emprendimiento basado en el conocimiento afecta positivamente el desempeño económico.

Desde otra posición surge una serie de desventajas como lo indica (Michelacci, 2003), en donde destaca de manera importante la falta de habilidades emprendedoras o empresariales, y subraya los esfuerzos que se deben hacer en el área de investigación y desarrollo para fomentar e incrementar los recursos al emprendimiento para crear capacidad empresarial al igual que capacidad en la investigación. Balmaceda (2018) y Michelacci (2003), mencionan otro enfoque exponiendo un panorama donde relacionan las habilidades con el área financiera, argumentan principalmente que las personas con una cartera equilibrada de habilidades tienden a mejorar aún más sus habilidades inherentes, lo que le permite fortalecerse en el aspecto del espíritu empresarial.

Por último, Balmaceda (2018), Hafer y Jones (2015) y Michelacci (2003) aportan información muy relevante sobre un cuestionamiento que revela el factor crucial que existe en el desarrollo de las habilidades cognitivas que permiten generar emprendedores de alta calidad y que, a su vez, crean y expanden negocios de alta calidad.

Otra de las contribuciones significativas la hace Poschke (2018), aludiendo las habilidades en tecnología empresarial. Justifica principalmente que el progreso en el campo técnico favorece mejores emprendedores. Con la colaboración de Brixiova et al. (2015) se desarrolló un modelo de estudio que se arraiga a las limitaciones que confronta el espíritu emprendedor dentro de un contexto donde se manifiesta bajos ingresos, específicamente el aporte que hacen estos investigadores es que en algunos países de bajos ingresos, el emprendimiento de oportunidades y su contribución al crecimiento y la creación de empleos han sido limitados.

Así mismo, enfatizan que la atención al espíritu empresarial como motor del crecimiento y la productividad se han intensificado con el bajo crecimiento mundial y la desaceleración del comercio mundial. Brixiová et al. (2015) elaboraron un informe que consiste en cerrar una brecha en la literatura sobre emprendimiento y desarrollo mediante un modelo de creación de empresas con la implementación de diferentes habilidades entre empresarios, jóvenes y adultos. 


\section{- Sub tema 2: Habilidades equilibradas}

La red de citaciones del subtema 2 muestra 89 nodos (artículos), las cuales 10 nodos fueron objeto de análisis porque son investigaciones que se enfocan en lo importante que es aplicar diferentes habilidades o desarrollar habilidades equilibradas para un proceso emprendedor que implique el alcance de oportunidades de generará un negocio.

Este sub tema de las habilidades emprendedoras hace referencia a la teoría de Lazear (2004; 2005), donde propone que los emprendedores son personas con diferentes habilidades y no se especializan en algún campo. Por ejemplo, cuando un emprendedor quiere crear un restaurante probablemente deba conocer acerca de contabilidad, aspectos jurídicos para el manejo de alimentos o publicidad para promocionar su negocio. En este sentido, no necesariamente debe ser un chef experto en el área, pero si es necesario que conozca de cocina para poder conectar las necesidades de los clientes con la experticia del chef. Lazear se refiere a este tipo de características con la expresión "Jack-of-all-trades" resaltando las habilidades equilibradas que son necesarias en un emprendedor.

Debido al impacto de la Teoría de Lazear, aparecieron varios estudios soportando esta propuesta. El primer trabajo que aparece en esta sub área es el de Wagner (2003), donde prueba que existe una relación entre la decisión de ser empresario y contar con diferentes habilidades. Este estudio entrevistó a 33.633 personas de Alemania controlando variables como género y edad. Más adelante, el mismo autor realiza otra prueba con 12.000 personas enviando un cuestionario confirmando los mismos resultados de su investigación anterior (Wagner, 2006).

Dentro de la red de citaciones también aparecen dos estudios más recientes, el primero soporta la Teoría de Lazear contrario a la influencia de la teoría del capital humano y su papel en el emprendimiento (Stuetzer et al. 2013a). El segundo realiza el análisis con carreras tecnológicas llegando a los mismos resultados (Chen \& Thompson, 2016). En este sentido, se fortalece la propuesta de Lazear sobre las habilidades equilibradas de los emprendedores.

Finalmente, esta teoría también ha sido relacionada con el impacto en el crecimiento de la firma, la han aplicado en otros contextos y también ha sido cuestionada. Por ejemplo, Stuetzer et al. (2013b) muestra la relación del equilibrio en las habilidades y su relación positiva con el progreso de un emprendimiento. También prueba dos hipótesis, la primera es la relación de la inversión en nuevas habilidades y la segunda, si los emprendedores nacen con ellas.

La primera se refiere a la inversión que se hace para desarrollarlas. Los resultados mostraron que las dos hipótesis son válidas, los emprendedores desarrollan las habilidades y también nacen con ellas. Hsieh, Parker y Van Praag (2017) añaden la variable aversión al riesgo para determinar que una persona se vuelva emprendedora más un equilibrio en sus habilidades. Ellos resaltan que se necesita este elemento para empujar la decisión de convertirse en emprendedor.

Por otro lado, el trabajo de Moog et al. (2015) analizan a investigadores para determinar si se puede aplicar también en este entorno. Los resultados mostraron 
que también para los académicos es necesario contar con antecedentes en diversas disciplinas, el tiempo destinado para el emprendimiento y estar cerca a otros emprendedores para que tomen la decisión de crear un negocio. Adicionalmente, Bublitz, Fritsch y Wyrwich (2015) proponen que una ciudad de negocios compensa una deficiencia en este equilibrio de habilidades.

Finalmente, Åstebro \& Thompson (2011) proponen ampliar la idea de Jack-of-alltrades argumentando que si los emprendedores son personas generalistas tendrían una gran variedad de trabajos. En este estudio muestran que las personas con una experiencia en trabajos diversos tienden a tener menores ingresos que los especialistas. Es decir, identifican el riesgo o el costo de ser emprendedor, aunque en las conclusiones relacionan a los emprendedores con altos ingresos. Este estudio utilizó la palabra "hobos" que quiere decir trabajador viajero haciendo referencia a la inestabilidad de los trabajos de un emprendedor.

\section{- Sub tema 3: Habilidades sociales}

La red de co-citaciones correspondientes al subtema 3 muestra 88 nodos (artículos), los cuales 11 artículos fueron objeto de análisis ya que especifican que para progresar en un proceso de emprendimiento es muy útil implementar interacciones con los demás, es decir, entablar habilidades sociales para alcanzar objetivos ante situaciones sociales específicas.

Dentro de este subtema se da forma a la teoría correspondiente a las habilidades sociales. Baron y Markman (2000) analizaron cómo las habilidades sociales pueden mejorar el éxito de los emprendedores y plantea una comparación, desde un cuestionamiento, acerca de por qué algunos emprendedores son más exitosos que otros en la construcción de nuevas empresas. Concretamente, componen un concepto que aclara que las habilidades sociales de los empresarios son competencias específicas que les ayudan a interactuar y generar contactos personales de manera productiva con otros individuos.

Adicionalmente, Tocher et al. (2012) resaltan la importancia de las habilidades sociales para influir en las acciones de los stakeholders, amplían estos conceptos analizando la relación de estas actividades con el desempeño. Sin embargo, analizan la habilidad política (que es un componente de la competencia social). La habilidad política es la habilidad individual para influenciar las acciones de otros dentro de un ambiente de negocios. Esta habilidad está positivamente asociada al desempeño.

Tocher et al. (2012) y Ferris et al. (2005) comparten una idea desde un enfoque distinto relacionado con la importancia de las habilidades políticas en las organizaciones. Menciona principalmente la falta de características que le permiten a un individuo ejercer influencias orientadas al éxito tales como la "habilidad política". En su trabajo hace intentos exhaustivos para explorar completamente el contenido de la habilidad política para considerar su potencial en implicaciones multidimensionales. 
Conjuntamente, Cong, Dempsey y Xie (2017) aportan datos acerca de la relación de la literatura empresarial y el comportamiento de los emprendedores para la construcción de redes sociales. Posteriormente hace énfasis en cómo hacer uso de los recursos de la red social para aportar al mejoramiento del rendimiento empresarial. Zhan y Kim (2015) relacionan las habilidades políticas con la red social. Establecen que la red social está relacionada con el desempeño pero que todavía no se ha mostrado lo que genera esta red social.

En el tema del emprendimiento se generan muchos casos de estudio con el propósito de revelar su importancia. Cong et al. (2017) comparten un estudio de una empresa emprendedora en China cuyo propósito es contribuir a una teoría unificada hacia la orientación empresarial, con la finalidad de considerar que el vínculo entre la orientación empresarial y el desempeño de una empresa depende de las habilidades políticas y de la relación con la justicia de una organización.

Desde otro punto de vista, Al Mamun, Fazal y Muniady (2019) aportan otro estudio concerniente a la innovación y emprendimiento de Asia Pacífico. Particularmente es un estudio que se realizó para determinar el conocimiento empresarial, habilidades, competencias y desempeños de microempresas en Kelantan, Malaysia. Hacen principal hincapié en promover e impulsar el espíritu empresarial para incrementar el número de emprendedores que tengan la iniciativa de crear empresas y así aportar al crecimiento de empleo. Al mismo tiempo describen la intención de fortalecer las habilidades de enfrentar muchos desafíos en ambientes de incertidumbre dentro de un mercado, capital humano y financiero y aumento de competidores locales e internacionales.

Finalmente, Baron y Tang (2009) muestran que algunas habilidades sociales (percepción social y expresividad) están relacionadas con el desempeño. Lo novedoso es que el estudio fue hecho en China. Para dar más importancia a lo descrito, Baron y Markman (2003), diseñan las preguntas para crear el constructo de competencia social. Definen que la competencia social, la habilidad para interactuar efectivamente con otros, está basada en habilidades sociales discretas.

Los resultados mostraron que un aspecto de la competencia social (precisión para percibir a otros) está relacionada positivamente con el éxito financiero del emprendedor. Por último, Huang, Frideger y Pearce (2013) evalúan el acento y las habilidades políticas para la adquisición de nuevas inversiones para el emprendimiento. Los resultados mostraron que el acento influye negativamente en este proceso. Lo importante es que analizaron también la raza, las habilidades en comunicación y colaboración, encontrando que estas no tienen mayor significancia.

\section{Conclusiones}

Mediante un análisis que se logró hacer a través de una revisión de literatura, con el uso de la plataforma Web of Science y la herramienta Tree of Science, donde este último presenta la información en forma de árbol y fue una fuente para adquirir la información conveniente para este documento el cual muestra la importancia que tiene el emprendimiento y las habilidades requeridas para iniciar una acción 
emprendedora. El tema del emprendimiento es importante para las personas con iniciativa de alcanzar una vida exitosa, por este motivo este artículo menciona y detalla algunas habilidades que permiten conseguir resultados satisfactorios en el desempeño emprendedor, es por eso que el enfoque del emprendimiento tiende a tener una estrecha relación con el desarrollo de habilidades y la exploración de oportunidades.

Gracias a los diversos estudios e investigaciones, permitieron que el término del emprendimiento ampliará su marco conceptual lo que significa que el estudio del espíritu emprendedor ha adquirido importancia y relevancia en las últimas décadas. El amplio marco conceptual que se le ha proporcionado al emprendimiento involucra información obtenida de muchos puntos de vista disciplinarios.

Además, los resultados abstractos acerca del emprendimiento, se han transformado en un punto de partida para ayudar a los investigadores a reconocer multitud de elementos necesarios y factores que componen el espíritu emprendedor que hacen avanzar la calidad del trabajo empírico y teórico en el campo. Igualmente, los artículos que fueron objeto de estudio permitieron evidenciar que existe una notoria ausencia de habilidades en cierto conjunto de comunidades que impiden que tengan una iniciativa emprendedora, y que para dar solución a esta situación, se propone generar niveles de educación que promuevan las destrezas de emprendimiento que conlleven a la búsqueda de oportunidades.

El resultado de la presente investigación se muestra empleando la analogía de la estructura de un árbol lo que permite poner de manera explícita la evolución de importancia de esta área de conocimiento. Los artículos de la raíz fueron considerados como la relación cercana que tiene el emprendimiento con la palabra de empresarios, básicamente el enfoque de la similitud que conservan ambos términos; los del tronco brindan información que le dan forma a la teoría del emprendimiento y las habilidades; y los de las hojas estiman una perspectiva global y escenarios internacionales.

Dentro de los resultados se evidencia que el enfoque del emprendimiento, en particular, abarca el descubrimiento y aprovechamiento de oportunidades rentables en donde es necesario aplicar habilidades indispensables que todo emprendedor potencial necesita tener para buscar nuevas fuentes de innovación, utilizar herramientas de creatividad y así emprender a la generación de organizaciones que se inserten en el mercado.

Se encontraron tres subtemas de las habilidades emprendedoras, el primer subtema analiza el impacto de las habilidades emprendedoras en la economía; el segundo subtema, muestra las habilidades equilibradas; y el tercer subtema, expone las habilidades sociales.

Respecto al nivel de importancia tan significativo que ha tenido y tiene las habilidades emprendedoras, los autores citados llegan a la conclusión de que el emprendimiento o espíritu empresarial son un método de acción humana, dicho de otra manera, es una forma influyente de abordar problemas grandes y permanentes dentro de las nuevas revoluciones que surgen actualmente. 
Es importante reconocer que el emprendimiento asume distintas definiciones en cuanto a su evolución, entre ellas está que se puede entender como un sistema individual o colectivo que son internos o externos a la estructura de una organización y que para ello, es preciso analizar e identificar habilidades, el cual es un término que históricamente conserva una importancia significativa debido a factores tecnológicos, organizativos y económicos y que de igual manera se ha utilizado para referirse a características individuales. Básicamente las habilidades se adoptan como una forma para preparar a los individuos o personas para crear un espíritu emprendedor.

$\mathrm{Al}$ igual que en cualquier tipo de investigación, este artículo presenta ciertas limitaciones. Como primer punto solo se empleó la base de datos web of science para hacer la búsqueda inicial. Y como segundo punto, el criterio de búsqueda de "entrepreneurship and skill" podría implicar ciertas limitaciones, al poder excluir palabras clave relacionadas con el tema.

Se sugiere para futuras investigaciones realizar un metanálisis de campo, también, de dar mayor profundidad a estudiar distintos enfoques de habilidades a los que están propuestas en este estudio, incluso se podría desarrollar un análisis utilizando otras herramientas diferentes a las usadas en este estudio para verificar la calidad la red de los subtemas propuesta. Adicionalmente, se sugiere utilizar el nuevo algoritmo de ToS para mejorar los resultados en las hojas (Valencia-Hernandez, Robledo, Pinilla, Duque-Méndez \& Olivar-Tost, 2020).

\section{REFERENCIAS}

Al Mamun, A., Fazal, S. A. \& Muniady, R. (2019). Entrepreneurial knowledge, skills, competencies and performance: A study of micro-enterprises in Kelantan, Malaysia. Asia Pacific Journal of Innovation and Entrepreneurship, 13(1), 29-48. https://doi.org/10.1108/APJIE-11-2018-0067

Åstebro, T. \& Thompson, P. (2011). Entrepreneurs, Jacks of all trades or Hobos? Research policy, 40(5), 637-649. https://doi.org/10.1016/j.respol.2011.01.010

Audretsch, D. B., Bönte, W. \& Keilbach, M. (2008). Entrepreneurship capital and its impact on knowledge diffusion and economic performance. Journal of Business Venturing, 23(6), 687-698. https://doi.org/10.1016/j.jbusvent.2008.01.006

Balmaceda, F. (2018). Entrepreneurship: skills and financing. Small Business Economics, 50(4), 871-886. https://doi.org/10.1007/s11187-017-9915-1

Baron, R. A. \& Tang, J. (2009). Entrepreneurs' Social Skills and New Venture Performance: Mediating Mechanisms and Cultural Generality. Journal of management, 35(2), 282-306. https://doi.org/10.1177/0149206307312513

Baron, R. A. \& Markman, G. D. (2003). Beyond social capital: the role of entrepreneurs' social competence in their financial success. Journal of Business Venturing, 18(1), 41-60. https://doi.org/10.1016/S0883-9026(00)00069-0

Baron, R. A. \& Markman, G. D. (2000). Beyond social capital: How social skills can enhance entrepreneurs' success. Academy of Management Perspectives, 14(1), 106-116. https://doi.org/10.5465/ame.2000.2909843 
Baumol, W. J. (1993). Formal entrepreneurship theory in economics: Existence and bounds. Journal of Business Venturing, 8(3), 197-210. https://doi. org/10.1016/0883-9026(93)90027-3

Bernat, T., Korpy, J., Grundey, D., Šavria, B., Bilan, Y. \& Koren, A. (2009). Researching students'entrepreneurship skills in post-socialist countries: a multicountry survey (Part 2). Transformations in Business \& Economics, 8(3), 104130. Disponible en http://www.transformations.knf.vu.lt/18/article/rese

Blondel, V. D., Guillaume, J.-L., Lambiotte, R. \& Lefebvre, E. (2008). Fast unfolding of communities in large networks. Journal of Statistical Mechanics: Theory and Experiment, (10), 1-12. https://doi.org/10.1088/1742-5468/2008/10/P10008

Brixiová, Z., Ncube, M. \& Bicaba, Z. (2015). Skills and Youth Entrepreneurship in Africa: Analysis with Evidence from Swaziland. World Development, 67(C), 11-26. https://doi.org/10.1016/j.worlddev.2014.09.027

Brixiova, Z. \& Égert, B. (2017). Entrepreneurship, institutions and skills in lowincome countries. Economic modelling, 67(1), 381-391. https://doi.org/10.1016/j. econmod.2017.02.020

Bublitz, E., Fritsch, M. \& Wyrwich, M. (2015). Balanced Skills and the City: An Analysis of the Relationship between Entrepreneurial Skill Balance, Thickness, and Innovation. Economic Geography, 91(4), 475-508. https://doi.org/10.1111/ ecge. 12097

Buitrago, S., Duque, P. L. \& Robledo, S. (2019). Branding Corporativo: una revisión bibliográfica. Económicas CUC, 41(1), 143-162. https://doi.org/10.17981/econcuc.41.1.2020.Org.1

Chen, C. C., Greene, P. G. \& Crick, A. (1998). Does entrepreneurial self-efficacy distinguish entrepreneurs from managers? Journal of Business Venturing, 13(4), 295-316. https://doi.org/10.1016/S0883-9026(97)00029-3

Chen, L.-W. \& Thompson, P. (2016). Skill Balance and Entrepreneurship Evidence from Online Career Histories. Entrepreneurship Theory and Practice, 40(2), 289-305. https://doi.org/10.1111/etap.12220

Cong, C., Dempsey, M. \& Xie, H. M. (2017). Political skill, entrepreneurial orientation and organizational justice: A study of entrepreneurial enterprise in China. International Journal of Entrepreneurial Behavior \& Research, 23(1), 20-34. https://doi.org/10.1108/IJEBR-05-2015-0103

Davidsson, P. \& Honig, B. (2003). The role of social and human capital among nascent entrepreneurs. Journal of Business Venturing, 18(3), 301-331. https://doi. org/10.1016/S0883-9026(02)00097-6

Díez-Gómez, D., Guillén, M. y Rodríguez, M. (2019). Revisión de la Literatura sobre la Toma de Decisiones Éticas en Organizaciones. Información Tecnológica, 30(3), 25-38. http://dx.doi.org/10.4067/S0718-07642019000300025

Duque, P. \& Cervantes-Cervantes, L.-S. (2019). Responsabilidad Social Universitaria: una revisión sistemática y análisis bibliométrico. Estudios Gerenciales, 35(153), 451-464. https://doi.org/10.18046/j.estger.2019.153.3389 
Ferris, G. R., Treadway, D. C., Kolodinsky, R. W., Hochwarter, W. A., Kacmar, C. J., Douglas, C. \& Frink, D. D. (2005). Development and Validation of the Political Skill Inventory. Journal of management, 31(1), 126-152. https://doi. org/10.1177/0149206304271386

Hafer, R. W. \& Jones, G. (2015). Are entrepreneurship and cognitive skills related? Some international evidence. Small Business Economics, 44(2), 283-298. https://doi.org/10.1007/s11187-014-9596-y

Hartog, J., Van Praag, M. \& Van Der Sluis, J. (2010). If You Are So Smart, Why Aren't You an Entrepreneur? Returns to Cognitive and Social Ability: Entrepreneurs Versus Employees. Journal of Economics \& Management Strategy, 19(4), 947-989. https://doi.org/10.1111/j.1530-9134.2010.00274.x

Henríquez, G., Rada, J. \& Torrenegra, A. (2016). Medición de variables psicológicas, económicas y sociales para identificar factores ocultos de los emprendedores en Barranquilla. Económicas CUC, 37(1), 179-202. https://doi.org/10.17981/econcuc.37.1.2016.08

Hodges, N., Watchravesringkan, K., Yurchisin, J., Hegland, J., Karpova, E., Marcketti, S. \& Yan, R.-N. T. (2015). Assessing Curriculum Designed to Foster Students' Entrepreneurial Knowledge and Small Business Skills from a Global Perspective. Family and consumer sciences research journal / American Association of Family and Consumer Sciences, 43(4), 313-327. https://doi.org/10.1111/ fcsr. 12115

Hsieh, C., Parker, S. C. \& Van Praag, C. M. (2017). Risk, balanced skills and entrepreneurship. Small Business Economics, 48(2), 287-302. https://doi.org/10.1007/ s11187-016-9785-y

Huang, L., Frideger, M. \& Pearce, J. L. (2013). Political skill: explaining the effects of nonnative accent on managerial hiring and entrepreneurial investment decisions. The Journal of Applied Psychology, 98(6), 1005-1017. https://doi. org/10.1037/a0034125

Krieger, A., Block, J. \& Stuetzer, M. (2018). Skill variety in entrepreneurship: A literature review and research directions. International Review of Entrepreneurship, 16(1), 29-62. Available from https://mpra.ub.uni-muenchen.de/88389/1/ MPRA_paper_88389.pdf

Kuratko, D. F. (2005). The Emergence of Entrepreneurship Education: Development, Trends, and Challenges. Entrepreneurship Theory and Practice, 29(5), 577-598. https://doi.org/10.1111/j.1540-6520.2005.00099.x

Landínez, D. A. \& Montoya, D. A. (2019). Políticas de salud pública para la prevención y el tratamiento de la enfermedad vascular cerebral: una revisión sistemática por medio de la metodología ToS (Tree of Science). Medicina UPB, 38(2), 129-139. https://doi.org/10.18566/medupb.v38n2.a05

Landínez, D. A., Robledo, S. \& Montoya, D. M. (2019). Executive Function performance in patients with obesity: A systematic review. Psychologia, 13(2), 121134. https://doi.org/10.21500/19002386.4230 
Lazear, E. P. (2005). Entrepreneurship. Journal of Labor Economics, 23(4), 649680. https://doi.org/10.1086/491605

Lazear, E. P. (2004). Balanced Skills and Entrepreneurship. The American Economic Review, 94(2), 208-211. https://doi.org/10.1257/0002828041301425

Mamabolo, A. \& Myres, K. (2020). A systematic literature review of skills required in the different phases of the entrepreneurial process. Small Enterprise Research, 5(1), 1-25. https://doi.org/10.1080/13215906.2020.1730230

Michelacci, C. (2003). Low Returns in R\&D due to the Lack of Entrepreneurial Skills. The Economic journal of Nepal, 113(484), 207-225. https://doi.org/10.1111/14680297.00095

Moog, P., Werner, A., Houweling, S. \& Backes-Gellner, U. (2015). The impact of skills, working time allocation and peer effects on the entrepreneurial intentions of scientists. The Journal of Technology Transfer, 40(3), 493-511. https:// doi.org/10.1007/s10961-014-9347-x

Oosterbeek, H., Van Praag, M. \& Ijsselstein, A. (2010). The impact of entrepreneurship education on entrepreneurship skills and motivation. European Economic Review, 54(3), 442-454. https://doi.org/10.1016/j.euroecorev.2009.08.002

Poschke, M. (2018). The Firm Size Distribution across Countries and Skill-Biased Change in Entrepreneurial Technology. American Economic Journal: Macroeconomics, 10(3), 1-41. https://doi.org/10.1257/mac.20140181

Reynolds, P., Bosma, N., Autio, E., Hunt, S., De Bono, N., Servais, I., Lopez-Garcia, P. \& Chin, N. (2005). Global Entrepreneurship Monitor: Data Collection Design and Implementation 1998-2003. Small Business Economics, 24(3), 205-231. https://doi.org/10.1007/s11187-005-1980-1

Robledo-Giraldo, S., Duque-Méndez, N. D. \& Zuluaga-Giraldo, J. I. (2013). Difusión de productos a través de redes sociales: una revisión bibliográfica utilizando la teoría de grafos. Respuestas, 18(2), 28-42. https://doi. org/10.22463/0122820x.361

Sabar, G. \& Pagis, M. (2015). Enhancing the spirit of entrepreneurship: African labor migrants returning from Israel. Migration Studies, 3(2), 260-280. https:// doi.org/10.1093/migration/mnu045

Sahlberg, P. (2006). Education Reform for Raising Economic Competitiveness. Journal of Educational Change, 7(4), 259-287. https://doi.org/10.1007/s10833-0054884-6

Sarasvathy, S. D. \& Venkataraman, S. (2011). Entrepreneurship as Method: Open Questions for an Entrepreneurial Future. Entrepreneurship Theory and Practice, 35(1), 113-135. https://doi.org/10.1111/j.1540-6520.2010.00425.x

Sousa, M. (2018). Entrepreneurship Skills Development in Higher Education Courses for Teams Leaders. Administrative Sciences, 8(2), 1-15. https://doi. org/10.3390/admsci8020018 
Stuetzer, M., Obschonka, M., Davidsson, P. \& Schmitt-Rodermund, E. (2013a). Where do entrepreneurial skills come from? Applied Economics Letters, 20(12), 1183-1186. https://doi.org/10.1080/13504851.2013.797554

Stuetzer, M., Obschonka, M. \& Schmitt-Rodermund, E. (2013b). Balanced skills among nascent entrepreneurs. Small Business Economics, 41(1), 93-114. https:// doi.org/10.1007/s11187-012-9423-2

Tocher, N., Oswald, S. L., Shook, C. L. \& Adams, G. (2012). Entrepreneur political skill and new venture performance: Extending the social competence perspective. Entrepreneurship and Regional Development, 24(5-6), 283-305. https://doi. org/10.1080/08985626.2010.535856

Valencia-Hernandez, D. S., Robledo, S., Pinilla, R., Duque-Méndez, N. D. \& OlivarTost, G. (2020). SAP Algorithm for Citation Analysis: An improvement to Tree of Science. Ingeniería e Investigación, 40(1), 45-49. https://doi.org/10.15446/ing. investig.v40n1.77718

Wagner, J. (2006). Are nascent entrepreneurs "Jacks-of-all-trades"? A test of Lazear's theory of entrepreneurship with German data. Applied Economics, 38(20), 2415-2419. https://doi.org/10.1080/00036840500427783

Wagner, J. (2003). Testing Lazear's jack-of-all-trades view of entrepreneurship with German micro data. Applied Economics Letters, 10(11), 687-689. https://doi. org/10.1080/1350485032000133273

Zhan, X. \& Kim, C.-K. (2015). A study on the political skill of Korean entrepreneurs contributing to social network guarantee. Nankai Business Review International, 6(3), 335-346. https://doi.org/10.1108/NBRI-11-2014-0043

Zuluaga, M., Robledo, S., Osorio-Zuluaga, G., Yathe, L., González, D. \& Taborda, G. (2016). Metabolomics and pesticides: Systematic literature review using graph theory for analysis of references. Nova, 14(25), 121-138. https://doi. org/10.22490/24629448.1735

BIODATA

Carolina Ocampo Sanabria es profesional en Negocios Internacionales de la Universidad Católica Luis Amigó (Colombia). https://orcid.org/0000-0002-2068-9701

Yurani López Espitia es Administradora de Empresas de la Universidad Nacional (Manizales, Colombia), Magister en Gerencia de Talento Humano de la Universidad de Manizales (Colombia) y Estudiante de Doctorado en Administración de la Universidad de Manizales. Coordinadora de los programas de Administración de Empresas y Negocios Internacionales de la Universidad Católica Luis Amigó (Manizales, Colombia). https://orcid.org/0000-0003-2757-7806 